\title{
Resveratrol ameliorates inflammatory damage and protects against osteoarthritis in a rat model of osteoarthritis
}

\author{
YULONG WEI ${ }^{*}$, JIE JIA* ${ }^{*}$ XIN JIN, WEI TONG and HONGTAO TIAN \\ Department of Orthopedics, Union Hospital, Tongji Medical College, Huazhong \\ University of Science and Technology, Wuhan, Hubei 430022, P.R. China
}

Received December 8, 2016; Accepted July 13, 2017

DOI: $10.3892 / \mathrm{mmr} .2017 .8036$

\begin{abstract}
Resveratrol is a non-flavonoid polyphenol compound with a stilbene structure. As a type of phytoalexin produced under stress in plants, it improves the plant's resistance against pathogens and environment deterioration, and performs important functions beneficial to human health, such as anti-cancer, anti-oxidation, regulating blood lipid levels and prolonging life span. The effects of resveratrol were examined in a rat model of osteoarthritis (OA) and observed to ameliorate inflammatory damage and protect against OA. In the present study, resveratrol significantly inhibited the induction of clinical scores in rats with OA. Resveratrol inhibited tumor necrosis factor- $\alpha$, interleukin (IL)-1 $\beta$, IL-6 and IL-18 expression levels, and decreased caspase-3/9 activity in rats with OA. Inducible nitric oxide synthase, nuclear factor $(\mathrm{NF})-\kappa \mathrm{B}$, phosphorylated-(p)-AMP-activated protein kinase and sirtuin 1 protein expression were significantly suppressed and heme oxygenase 1 (HO-1) and nuclear factor erythroid 2-related factor 2 (Nrf-2) protein expression was stimulated in rats with OA treated with resveratrol. The current results indicate that resveratrol ameliorates inflammatory damage and protects against $\mathrm{OA}$ in a rat model of $\mathrm{OA}$ via $\mathrm{NF}-\kappa \mathrm{B}$ and $\mathrm{HO}-1 / \mathrm{Nrf}-2$ signaling.
\end{abstract}

\section{Introduction}

Osteoarthritis (OA) is a common chronic degenerative joint disease, primarily characterized by degradation of cartilage, synovitis and subchondral bone remodeling, it is a disease that affects all joints in the body (1). The epidemiological survey

Correspondence to: Dr Hongtao Tian, Department of Orthopedics, Union Hospital, Tongji Medical College, Huazhong University of Science and Technology, 1277 JieFang Road, Wuhan, Hubei 430022, P.R. China

E-mail: tongnqitlr@163.com

*Contributed equally

Key words: resveratrol, osteoarthritis, inflammatory, nuclear factor- $\kappa \mathrm{B}$, heme oxygenase 1/nuclear factor erythroid 2-related factor 2 did not identify that OA had obvious racial and regional differences (1). Arm arthritis accounts for $2-6 \%$ of disabilities in people aged $>50$ years, only secondary to vascular diseases in terms of long-term disability (2). Due to high morbidity and disability rates in the elderly, OA is considered to be one of the most common diseases affecting human health (3).

Nuclear factor $(\mathrm{NF})-\kappa \mathrm{B}$, a nuclear factor that specifically binds to the $\kappa \mathrm{B}$ sequence of immunoglobulin $\mathrm{K}$ chain gene enhancer, is the central transcription factor, which participates in inflammation and the immune response. It is activated by interleukin (IL)-1 $\beta$, tumor necrosis factor (TNF)- $\alpha$ and other cytokines, and rapidly induces the expression of multiple genes, which participate in immune reactions and the inflammatory response via a series of reactions, such as cytokine TNF- $\alpha$, IL-1 $\beta$, IL- 6 and IL-18, inflammatory enzyme and matrix metalloproteinase (MMPs). Previous studies demonstrated that the $\mathrm{NF}-\kappa \mathrm{B}$ signaling pathway was activated in a rat model of $\mathrm{OA}(4,5)$.

The imbalance of the oxidation-reduction reaction leads to the accumulation of reactive oxygen species in the human body, which eventually results in oxidative stress (6). Under oxidative stress, large-molecular substances, such as proteins, lipids, DNA and RNA, and particularly damaged protein, lose their normal functions and their structures are impaired by oxidative damage (7).

Chondrocytes secrete more proinflammatory cytokines due to pathological factors, among which TNF- $\alpha$ and IL-1 $\beta$ perform the most important roles. These proinflammatory cytokines promote the formation of MMPs and speed up the extracellular matrix degradation of chondrocytes, significantly contributing to the destruction of the articular cartilage (8). Due to congestion, edema and the aggregation of inflammatory mediators, synovial inflammation induces synovial cells to secrete a variety of proteases and proinflammatory cytokines. Proteases, such as MMPS, contribute to degradation of the articular cartilage. Proinflammatory cytokines, such as TNF- $\alpha$, IL-1 $\beta$, IL- 6 and IL-18 act on synovial cells and chondrocytes to increase the secretion of MMPS, and inhibit the synthesis of proteoglycan and collagen (9).

Resveratrol was first extracted from the root of Veratrum grandiflorum by a Japanese man in 1940 (10). Resveratrol is the active constituent of certain herbal medicines that treat inflammation, lipid metabolism disorder and heart diseases (11). As an important secondary metabolite of 
plants, resveratrol contributes to health care and a variety of important biological functions (12). Resveratrol protects the cardiovascular system by reducing myocardial ischemia reperfusion injury, dilating blood vessels and preventing against atherosclerosis (13). As resveratrol dilates blood vessels, it lowers blood pressure and reduces the risk of cardiovascular diseases (13). People have long used Polygonum cuspidatum, Polygonum multiflorum and other traditional Chinese medicines rich in resveratrol to treat and prevent against hyperlipidemia and atherosclerosis (13). Recent studies have also indicated that resveratrol is an effective constituent preventing against cardiac dysfunction $(13,14)$. Thus, the aim of the present study was to determine the effects of resveratrol on inflammatory damage in a rat model of OA, and its underlying mechanism.

\section{Materials and methods}

Animals. All animal procedures were approved by the Animal Research Ethics Committee at Tongji Medical College (Wuhan, China). Male Wistar rats (age, 6 weeks, $n=30$ ), weighing 200-230 g, were purchased from Central Lab of Tongji Medical College (Wuhan, China). Animals were maintained at Tongji Medical College (Tongji, China) and housed three per cage in a room with controlled temperature conditions $\left(21-22^{\circ} \mathrm{C}\right)$, controlled lighting (12-h light/dark cycle) and access to sterile food and water.

Induction of the OA model rat and grouping. The animals were randomized and assigned to three groups $(n=10 /$ per group) as follows: Sham group, OA model group and resveratrol treatment group. Following anesthetization with isoflurane (Sigma-Aldrich; Merck KGaA, Darmstadt, Germany), rats in the OA model group and the resveratrol treatment group were injected with intra-articular injection of monosodium iodoacetate (Sigma-Aldrich; Merck KGaA) through the patellar ligament into the intra-articular space of the right knee. Rats in the resveratrol treatment group were administered with $50 \mathrm{mg} / \mathrm{kg} / 3$ days resveratrol, for 8 weeks. Rats were sacrificed using decollation under $35 \mathrm{mg} / \mathrm{kg}$ pentobarbital sodium (Sigma-Aldrich; Merck KGaA), after treatment with resveratrol.

Clinical score of $O A$. A total score of 16 was recorded for each rat. The clinical score was measured as follows: 0 , No swelling; $1, \geq 0.1 \mathrm{~mm}$ increase in paw swelling or 1 digit; 2 , $\geq 0.2 \mathrm{~mm}$ increase in paw swelling and two groups of inflamed joints; $3, \geq 0.3 \mathrm{~mm}$ increase in paw swelling and two groups of inflamed joints; $4, \geq 0.45 \mathrm{~mm}$ increase in paw swelling and two groups of inflamed joints (15).

Western blot analysis. Joint tissue samples were acquired from rats and washed with PBS. Total protein of the tissue samples was extracted using a protein radioimmunoprecipitation assay (Beyotime Institute of Biotechnology, Nantong, China) and Protein concentrations were measured using a Bicinchoninic Protein Assay kit (Beyotime Institute of Biotechnology). Equal quantities $(50 \mu \mathrm{g})$ of total protein were separated by electrophoresis $(80 \mathrm{~V}$ for $30 \mathrm{~min}$ and $120 \mathrm{~V}$ for $1 \mathrm{~h})$ on $12 \%$ SDS-PAGE and electrophoretically transferred

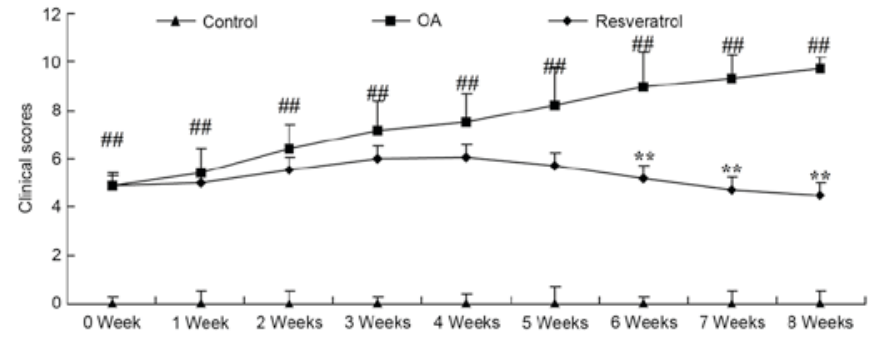

Figure 1. Resveratrol ameliorates clinical scores in OA rats. Control, control group; OA, OA model group; Resveratrol, resveratrol treatment group. ${ }^{\# \#} \mathrm{P}<0.01$ vs. control group, ${ }^{* *} \mathrm{P}<0.01$ vs. OA model group. OA, osteoarthritis.

onto polyvinylidene difluoride membranes. Membranes were blocked with $5 \%$ skimmed milk in tris buffered saline with $0.1 \%$ Tween for $1 \mathrm{~h}$ at $37^{\circ} \mathrm{C}$ and incubated with $\mathrm{NF}-\kappa \mathrm{B}$ (cat. no. sc-56735, 1:1,000; Santa Cruz Biotechnology, Inc., Dallas, TX, USA), p-AMP-activated protein kinase (AMPK; 1:1,000; Santa Cruz Biotechnology, Inc.), sirtuin 1 (SIRT1; cat. no. sc-15404,1:1,000; Santa Cruz Biotechnology, Inc.), heme oxygenase 1 (HO-1; cat. no. sc-10789, 1:1,000; Santa Cruz Biotechnology, Inc.), nuclear factor erythroid 2-related factor 2 (Nrf-2; cat. no. sc-722, 1:1,000; Santa Cruz Biotechnology, Inc.) and GAPDH (cat. no. sc-25778, 1:1,000; Santa Cruz Biotechnology, Inc.) at $4^{\circ} \mathrm{C}$ after the membranes were washed with Tris-buffered saline with Tween 20. Membranes were developed using an ECL detection system (Beyotime Institute of Biotechnology) subsequent to incubation with peroxidase-conjugated secondary antibodies (cat. no. sc-2004, 1:5,000; Santa Cruz Biotechnology, Inc.) at $37^{\circ} \mathrm{C}$ for $1 \mathrm{~h}$.

Statistical analysis. The results are presented as means \pm standard deviation. Statistical analysis was performed by one-way analysis of variance for multiple comparisons and $\mathrm{P}<0.05$ was considered to indicate a statistically significant difference.

\section{Results}

Resveratrol ameliorates the clinical scores in $O A$ rat. There was a significant increase of clinical scores in OA rats compared with the control group (Fig. 1; $\mathrm{P}<0.01$ ). Treatment with resveratrol significantly ameliorated the clinical scores in the OA rats when compared with the OA model (Fig. 1; $\mathrm{P}<0.01)$.

Resveratrol ameliorates TNF- $\alpha, I L-1 \beta, I L-6$ and $I L-18$ expression levels in $O A$ rats. To investigate the protection mechanism of resveratrol on OA rats, TNF- $\alpha$, IL-1 $\beta$, IL-6 and IL-18 expression levels were measured. As shown in Fig. 2, TNF- $\alpha$, IL-1 $\beta$, IL-6 and IL-18 expression levels in the OA rats were significantly increased compared with the control group (Fig. 2; $\mathrm{P}<0.01$ ). Treatment with resveratrol significantly ameliorated TNF- $\alpha$, IL-1 $\beta$, IL- 6 and IL-18 expression levels in $\mathrm{OA}$ rats compared with the $\mathrm{OA}$ model rats (Fig. 2; $\mathrm{P}<0.01$ ).

Resveratrol suppresses inducible nitric oxide synthase (iNOS), $N F-\kappa B, p-A M P K$ and SIRT1 protein expression levels in $O A$ rats. To investigate the anti-inflammation effect of resveratrol in OA rat, iNOS, NF- $\mathrm{B}$, p-AMPK and SIRT1 protein expression levels were analyzed using western blotting. As shown 


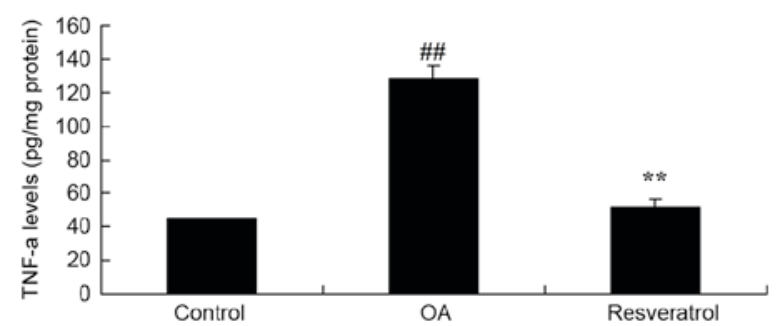

C

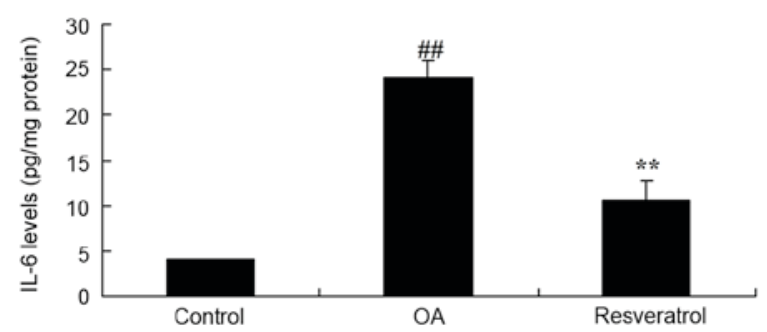

B

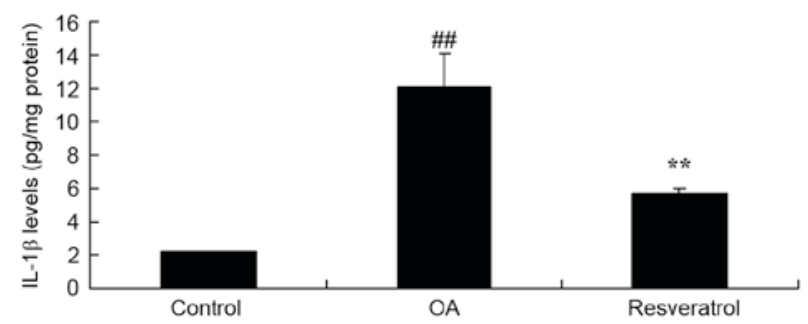

D

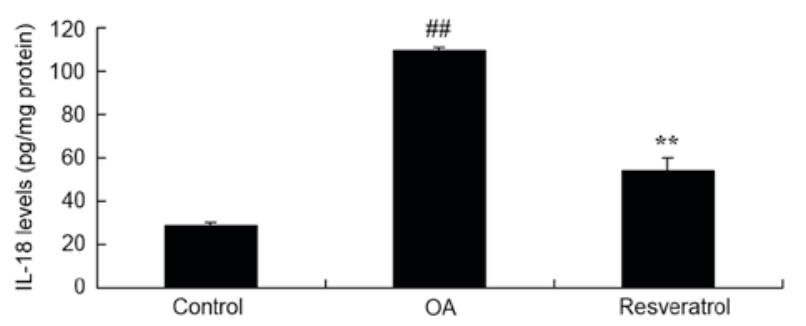

Figure 2. Resveratrol ameliorates TNF- $\alpha$, IL-1 $\beta$, IL-6 and IL-18 expression levels in OA rats. Resveratrol ameliorates (A) TNF- $\alpha$, (B) IL-1 $\beta$, (C) IL-6 and (D) IL-18 expression levels in OA rats Control, control group; OA, OA model group; Resveratrol, resveratrol treatment group. ${ }^{\#} \mathrm{P}<0.01$ vs. control group, ${ }^{* *} \mathrm{P}<0.01$ vs. OA model group. OA, osteoarthritis. TNF, tumor necrosis factor; IL, interleukin; OA, osteoarthritis.

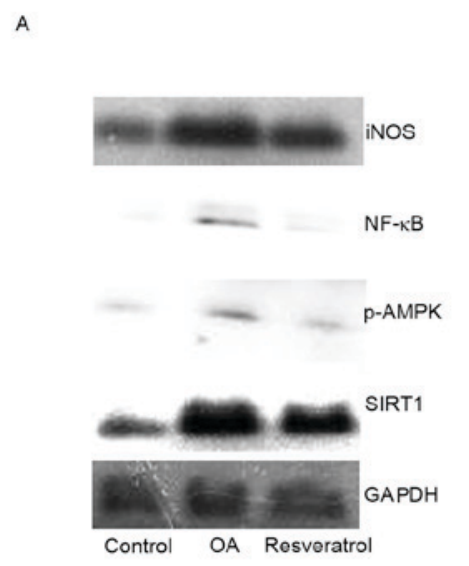

B
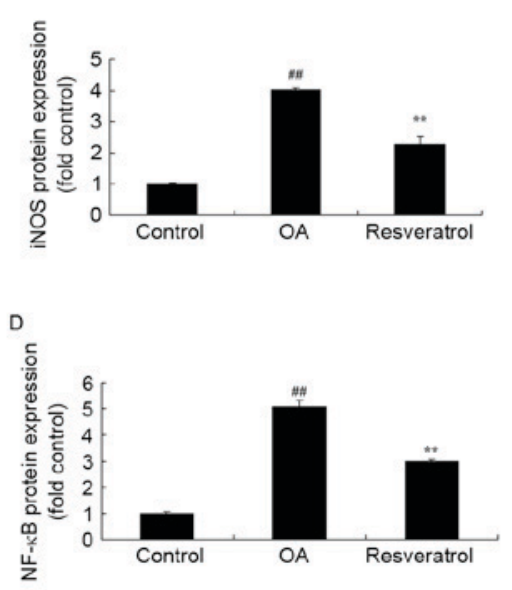
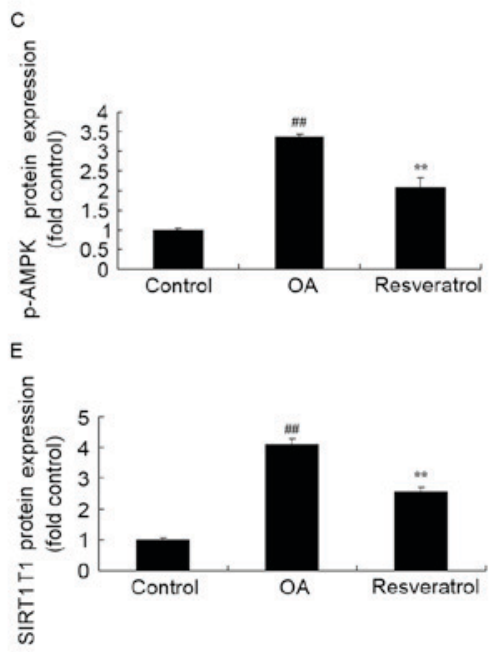

Figure 3. Resveratrol suppresses iNOS, NF-кB, p-AMPK and SIRT1 protein expression in OA rats. (A) Representative western blot images. Quantification of (B) iNOS, (C) p-AMPK, (D) NF- $\mathrm{B}$ and (E) and SIRT1 protein expression levels by resveratrol. Statistical analysis was performed following western blot analysis for iNOS, NF- $\kappa$ B, p-AMPK and SIRT1 protein expression in OA rats. ${ }^{\# \#} \mathrm{P}<0.01$ vs. control group, ${ }^{* *} \mathrm{P}<0.01$ vs. OA model group. OA, osteoarthritis; iNOS, inducible nitric oxide synthase; NF, nuclear factor; AMPK, AMP-activated protein kinase; SIRT1, sirtuin 1.

in Fig. 3, there were significant increases in iNOS, NF- $\kappa$ B, p-AMPK and SIRT1 protein expression levels in OA rats when compared with the control rats $(\mathrm{P}<0.01)$. Treatment with resveratrol significantly suppressed the increased levels of iNOS, NF- $\kappa$ B, p-AMPK and SIRT1 protein expression in the $\mathrm{OA}$ rats, compared with the OA models (Fig. 3; $\mathrm{P}<0.01$ ).

Resveratrol inhibits 3,4-methylenedioxyamphetamine (MDA) and superoxide dismutase (SOD) levels in OA rast. To further investigate the protection mechanism of resveratrol on OA rats, SOD and MDA levels were measured using ELISA kits. As presented in Fig. 4, OA effectively inhibited the SOD level and increased the MDA level in OA rats, when compared with the control group. Treatment with resveratrol significantly recovered the inhibited SOD level and reduced the increased MDA level in the OA rat, compared with the OA model (Fig. 4; $\mathrm{P}<0.01)$.

Resveratrol induces $\mathrm{HO}-1$ and Nrf-2 protein expression levels in $\mathrm{OA}$ rats. The effect of resveratrol on $\mathrm{HO}-1$ and Nrf-2 protein expression levels was evaluated in OA rats. As presented in Fig. 5, HO-1 and Nrf-2 protein expression levels were significantly lower in OA rats than in the control rats $(\mathrm{P}<0.01)$. Treatment with resveratrol significantly induced HO-1 and Nrf-2 protein expression levels in OA rats, compared with the OA model (Fig. 5; $\mathrm{P}<0.01$ ). 
A

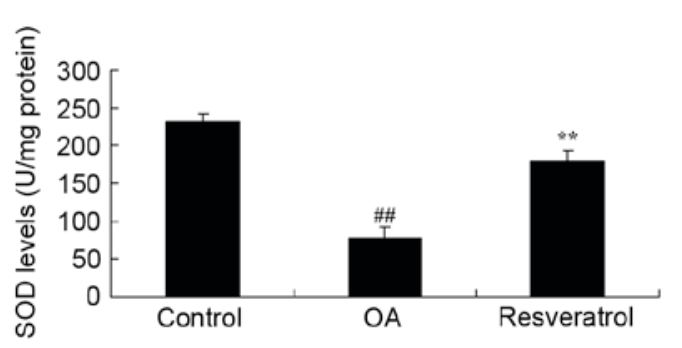

B

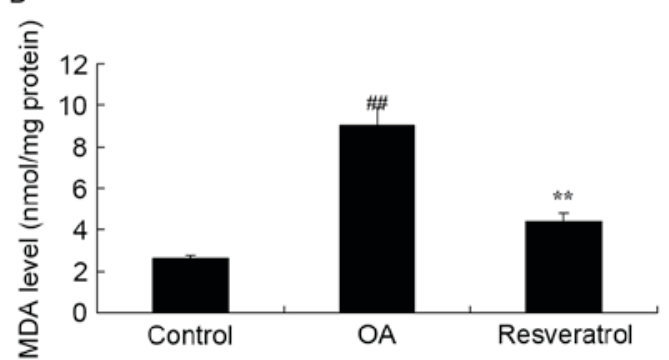

Figure 4. Resveratrol inhibits MDA and SOD levels in OA rats. Effects of resveratrol (A) inhibits MDA and (B) increases SOD levels in OA rats. Control, control group; OA, OA model group; Resveratrol, resveratrol treatment group. ${ }^{\# \#} \mathrm{P}<0.01$ vs. control group, ${ }^{* *} \mathrm{P}<0.01$ vs. OA model group. OA, osteoarthritis; MDA, 3,4-methylenedioxyamphetamine; SOD, superoxide dismutase.
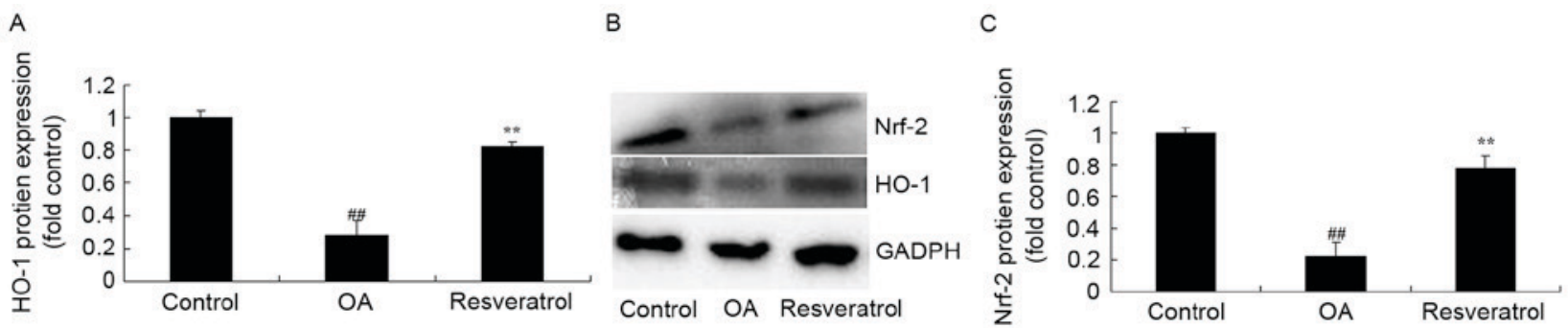

Figure 5. Resveratrol induces HO-1 and Nrf-2 protein expression in OA rats. (A) HO-1 protein expression. N (B) Representative western blot images. (C) Nrf-2 protein expression. Control, control group; OA, OA model group; Resveratrol, resveratrol treatment group. ${ }^{* \#} \mathrm{P}<0.01$ vs. control group, ${ }^{* * *} \mathrm{P}<0.01 \mathrm{vs}$. OA model group. OA, osteoarthritis; HO-1, heme oxygenase 1; Nrf-2, nuclear factor erythroid 2-related factor 2.

Resveratrol reduces caspase-3/9 activity in OA rats. The anti-apoptosis effects of resveratrol were investigated in OA rats. As shown in Fig. 6, OA effectively induced caspase-3/9 activity in the OA rat models compared with the control group. Treatment with resveratrol significantly reduced caspase-3/9 activity in OA rats compared with the OA model (Fig. 6; $\mathrm{P}<0.01)$.

\section{Discussion}

$\mathrm{OA}$, one of the most common chronic bone and joint degenerative diseases, may affect the joints of the limbs and spine, with pathological characteristics, including lesions in joint cartilage and subchondral bone (16). The clinical manifestations of OA primarily include pain, stiffness, deformation and limited movement of affected joints (17). Due to the aging population in China, the incidence of $\mathrm{OA}$ is increasing, with $50 \%$ of the population aged $>60$ years diagnosed with OA by X-ray, of which $35-50 \%$ present with clinical manifestations, and $80 \%$ of the population $>75$-years-old present with OA symptoms in China (18). In the current study, resveratrol treatment was found to ameliorate inflammatory damage, oxidative stress and neuronal apoptosis, and protect against OA in rat models of OA. This is consistent with Pan et al (12), who indicated that resveratrol protects against neonatal brain injury following hypoxia-ischemia via anti-apoptotic and anti-inflammatory effects (12).

The pathogenesis of OA remains unclear, although it has been confirmed that $\mathrm{NO}$ is significant in the incidence and development of OA (19). NO, an important regulatory factor participating in the differentiation and apoptosis of chondrocytes, inhibits the proliferation of chondrocytes and induces

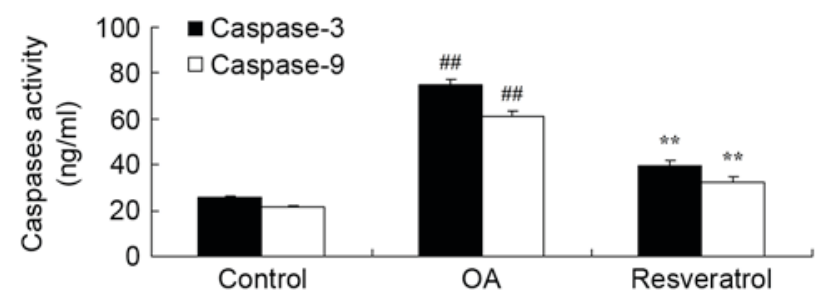

Figure 6. Resveratrol reduces caspase-3/9 activity in OA rats. Control, control group; OA, OA model group; Resveratrol, resveratrol treatment group. ${ }^{\# \#} \mathrm{P}<0.01$ vs. control group, ${ }^{* *} \mathrm{P}<0.01$ vs. OA model group. OA, osteoarthritis.

the apoptosis of chondrocytes. In addition, NO suppresses the synthesis of proteoglycan and type II collagen secreted by chondrocytes, and promotes the decomposition of cartilage extracellular matrix (20). NOS is the key enzyme inducing NO in patients with OA $(9,20)$. Furthermore, the current data indicates that resveratrol significantly suppressed the increased levels of iNOS protein expression in OA rats. Wang et al (19) reported that resveratrol ameliorates the integrity of the blood-brain barrier by maintaining iNOS expression.

Studies of AMPK predominantly focused on energy metabolism, and very few studies investigate the association between AMPK and inflammatory reactions $(21,22)$. However, increasing numbers of studies have demonstrated that AMPK exerted anti-inflammatory effects. For example, AMPK is pivotal in the regulation of energy metabolism in skeletal muscles, and is involved in energy metabolism and inflammation of skeletal muscles (23). In skeletal muscles, AMPK exerts anti-inflammatory effects by inhibiting the expression level 
of $N F-\kappa B(24)$. Activation of the $N F-\kappa B$ signaling pathway, a pro-inflammatory signaling pathway in the skeletal muscle cells of mice, may be inhibited by AMPK, and peroxisome proliferation activated receptor inhibits skeletal muscular inflammation and insulin resistance by inhibiting endoplasmic reticulum stress via the AMPK-dependent signaling pathway (22). In the present study, resveratrol significantly suppressed AMPK protein expression levels in OA rats. Meng et al (21) demonstrated that resveratrol attenuated estrogen deficient-induced cardiac dysfunction through enhancement of SIRT1/AMPK activity (21).

$\mathrm{NF}-\kappa \mathrm{B}$ is stimulated by various factors, such as TNFs and ILs (24). Activated NF- $\kappa$ B dissociates from its inhibitory proteins and translocates into the nucleus from the cytoplasm, and binds to specific sequences (at the $\kappa \mathrm{B}$ site) of promoter/enhancer, located upstream of the $\mathrm{NF}-\kappa \mathrm{B}$ signaling pathway, to adjust the expression levels of target genes $(19,24)$. The activated NF- $\kappa \mathrm{B}$ signaling pathway leads to the transcription of a series of downstream target genes, resulting in associated biological effects, such as the generation of inflammatory factors and the induction of chondrocyte apoptosis (10). Furthermore, resveratrol significantly reduced the level of $\mathrm{NF}-\kappa \mathrm{B}$ protein expression in OA rats.

SIRT1 is expressed in human articular chondrocytes and cartilage; however, its expression level in joint chondrocytes is lower than that in normal chondrocytes of patients with OA (25). In addition, it has been found that SIRT1 promotes the expression of cartilage specific genes, and enhances the anti-stress ability of chondrocytes, promotes cell survival and inhibits chondrocyte apoptosis, indicating that SIRT1 prevents against OA by inhibiting the aging and apoptosis of chondrocytes (26). These findings indicate that resveratrol significantly reduces SIRT1 protein expression levels in OA rats. Gan et al (27) demonstrated that resveratrol reduced acute kidney injury in septic rats via SIRT1 and de-acetylated NF-кB-P65 (27).

HO-1/Nrf-2 has been confirmed to act against oxidation, proliferation of malignant cells, apoptosis and inflammation, indicating that $\mathrm{HO}-1 / \mathrm{Nrf}-2$ is a protective molecule in the body and has been used for the treatment of various diseases (28). At present, studies indicate that HO-1 alleviates lupus nephritis; an experimental study with animal models of elastase-induced emphysema indicated that gene therapy with adenovirus vector-mediated $\mathrm{HO}-1 / \mathrm{Nrf}-2$ exerted a sound curative effect (29). In addition, HO-1/Nrf-2 gene-transfected chemical induction therapy and gene therapy are effective in the treatment of heart and liver injury induced by ischemia reperfusion, lipopolysaccharide-induced intraocular inflammation and cardiac allograft rejection (30). By contrast, studies on patients lacking $\mathrm{HO}-1 / \mathrm{Nrf}-2$ indicated that $\mathrm{HO}-1 / \mathrm{Nrf}-2$ deficiency may delay and worsen inflammation (31). Taken together, the current data suggests that resveratrol significantly reduced caspase-3/9 activity in OA rats. Cheng et al (32) reported that resveratrol attenuates inflammation and oxidative stress via the Nrf-2/antioxidant responsive element signaling pathway in myocardial ischemia-reperfusion injury (32).

In conclusion, the current study demonstrated that resveratrol ameliorates inflammatory damage, oxidative stress and neuronal apoptosis, and protects against OA in rat models of $\mathrm{OA}$, via further suppression of $\mathrm{NF}-\kappa \mathrm{B}$ and activation of HO-1/Nrf-2 signaling. Thus, resveratrol may present as an effective therapeutic strategy for patients with OA. However, this study only used a rat model, and therefore requires further study in other models or clinical trials.

\section{References}

1. Benazzo F, Perticarini L, Padolino A, Castelli A, Gifuni P, Lovato M, Manzini C and Giordan N: A multi-centre, open label, long-term follow-up study to evaluate the benefits of a new viscoelastic hydrogel (Hymovis ${ }^{\circledR}$ ) in the treatment of knee osteoarthritis. Eur Rev Med Pharmacol Sci 20: 959-968, 2016.

2. Rini C, Porter LS, Somers TJ, McKee DC, DeVellis RF, Smith M, Winkel G, Ahern DK, Goldman R, Stiller JL, et al: Automated Internet-based pain coping skills training to manage osteoarthritis pain: A randomized controlled trial. Pain 156: 837-848, 2015.

3. Schmal H, Salzmann GM, Langenmair ER, Henkelmann R, Südkamp NP and Niemeyer P: Biochemical characterization of early osteoarthritis in the ankle. ScientificWorldJournal 2014: 434802, 2014.

4. Scanzello CR: Chemokines and inflammation in osteoarthritis: Insights from patients and animal models. J Orthop Res 35: 735-739, 2017.

5. Cicuttini FM and Wluka AE: Not just loading and age: The dynamics of osteoarthritis, obesity and inflammation. Med J Aust 204: 47, 2016.

6. Hsu DZ, Chu PY and Jou IM: Daily sesame oil supplement attenuates joint pain by inhibiting muscular oxidative stress in osteoarthritis rat model. J Nutr Biochem 29: 36-40, 2016.

7. Li D, Xie G and Wang W: Reactive oxygen species: The 2-edged sword of osteoarthritis. Am J Med Sci 344: 486-490, 2012.

8. Fu Y, Lei J, Zhuang Y, Zhang K and Lu D: Overexpression of HMGB1 A-box reduced IL-1 $\beta$-induced MMP expression and the production of inflammatory mediators in human chondrocytes. Exp Cell Res 349: 184-190, 2016.

9. MaZ,Piao T, Wang Y and Liu J: Astragalin inhibits IL-1 $\beta$-induced inflammatory mediators production in human osteoarthritis chondrocyte by inhibiting NF- $\mathrm{B}$ and MAPK activation. Int Immunopharmacol 25: 83-87, 2015.

10. Aggarwal BB, Bhardwaj A, Aggarwal RS, Seeram NP, Shishodia S and Takada Y: Role of resveratrol in prevention and therapy of cancer: Preclinical and clinical studies. Anticancer Res 24: 2783-2840, 2004.

11. Wu C, Zhang Y, Shen Q, Zhou Z, Liu W and Hua J: Resveratrol changes spermatogonial stem cells (SSCs) activity and ameliorates their loss in busulfan-induced infertile mouse. Oncotarget 7: 82085-82096, 2016.

12. Pan S, Li S, Hu Y, Zhang H, Liu Y, Jiang H, Fang M, Li Z, Xu K, Zhang $\mathrm{H}$, et al: Resveratrol post-treatment protects against neonatal brain injury after hypoxia-ischemia. Oncotarget 7 : 79247-79261, 2016.

13. Gharaee-Kermani M, Moore BB and Macoska JA: Resveratrol-mediated repression and reversion of prostatic myofibroblast phenoconversion. PLoS One 11: e0158357, 2016.

14. Bai T, Hu X, Zheng Y, Wang S, Kong J and Cai L: Resveratrol protects against lipopolysaccharide-induced cardiac dysfunction by enhancing SERCA2a activity through promoting the phospholamban oligomerization. Am J Physiol Heart Circ Physiol 311: H1051-H1062, 2016.

15. Pan T, Chen R, Wu D, Cai N, Shi X, Li B and Pan J: Alpha-Mangostin suppresses interleukin-1 $\beta$-induced apoptosis in rat chondrocytes by inhibiting the NF- $\mathrm{KB}$ signaling pathway and delays the progression of osteoarthritis in a rat model. Int Immunopharmacol 52: 156-162, 2017.

16. Lomonte AB, de Morais MG, de Carvalho LO and Zerbini CA: Efficacy of triamcinolone hexacetonide versus methylprednisolone acetate intraarticular injections in knee osteoarthritis: A randomized, double-blinded, 24-week study. J Rheumatol 42: 1677-1684, 2015.

17. Pfitzner T, Abdel MP, von Roth P, Perka C and Hommel H: Small improvements in mechanical ax is alignment achieved with MRI versus CT-based patient-specific instruments in TKA: A randomized clinical trial. Clin Orthop Relat Res 472: 2913-2922, 2014.

18. Runhaar J, van Middelkoop M, Reijman M, Willemsen S, Oei EH, Vroegindeweij D, van Osch G, Koes B and Bierma-Zeinstra SM: Prevention of knee osteoarthritis in overweight females: The first preventive randomized controlled trial in osteoarthritis. Am J Med 128: 888-895.e4, 2015. 
19. Wang J, Kalhor A, Lu S, Crawford R, Ni JD and Xiao Y: iNOS expression and osteocyte apoptosis in idiopathic, non-traumatic osteonecrosis. Acta Orthop 86: 134-141, 2015.

20. Balaganur V, Pathak NN, Lingaraju MC, More AS Latief N, Kumari RR, Kumar D and Tandan SK: Effect of S-methylisothiourea, an inducible nitric oxide synthase inhibitor, in joint pain and pathology in surgically induced model of osteoarthritis. Connect Tissue Res 55: 367-377, 2014.

21. Meng Z, Jing H, Gan L, Li H and Luo B: Resveratrol attenuated estrogen-deficient-induced cardiac dysfunction: Role of AMPK, SIRT1, and mitochondrial function. Am J Transl Res 8: 2641-2649, 2016.

22. Tang CH, Chiu YC, Tan TW, Yang RS and Fu WM: Adiponectin enhances IL-6 production in human synovial fibroblast via an AdipoR1 receptor, AMPK, p38, and NF-kappa B pathway. J Immunol 179: 5483-5492, 2007.

23. Zhou Y, Liu SQ, Yu L, He B, Wu SH, Zhao Q, Xia SQ and Mei HJ: Berberine prevents nitric oxide-induced rat chondrocyte apoptosis and cartilage degeneration in a rat osteoarthritis mode via AMPK and p38 MAPK signaling. Apoptosis 20: 1187-1199, 2015.

24. Yan H, Duan X, Pan H, Holguin N, Rai MF, Akk A, Springer LE, Wickline SA, Sandell LJ and Pham CT: Suppression of NF-кB activity via nanoparticle-based siRNA delivery alters early cartilage responses to injury. Proc Natl Acad Sci USA 113: E6199-E6208, 2016.

25. Oh H, Kwak JS, Yang S, Gong MK, Kim JH, Rhee J, Kim SK, Kim HE, Ryu JH and Chun JS: Reciprocal regulation by hypoxia-inducible factor- $2 \alpha$ and the NAMPT-NAD(+)-SIRT axis in articular chondrocytes is involved in osteoarthritis Osteoarthritis Cartilage 23: 2288-2296, 2015.

26. Fu Y, Kinter M, Hudson J, Humphries KM, Lane RS, White JR, Hakim M, Pan Y, Verdin E and Griffin TM: Aging promotes sirtuin 3-dependent cartilage superoxide dismutase 2 acetylation and osteoarthritis. Arthritis Rheumatol 68: 1887-1898, 2016.
27. Gan Y, Tao S, Cao D, Xie H and Zeng Q: Protection of resveratrol on acute kidney injury in septic rats. Hum Exp Toxicol 36 1015-1022, 2017.

28. Rousset F, Nguyen MV, Grange L, Morel F and Lardy B: Heme oxygenase- 1 regulates matrix metalloproteinase MMP-1 secretion and chondrocyte cell death via Nox4 NADPH oxidase activity in chondrocytes. PLoS One 8: e66478, 2013.

29. Kyostio-Moore S, Bangari DS, Ewing P, Nambiar B, Berthelette P, Sookdeo C, Hutto E, Moran N, Sullivan J, Matthews GL, et al: Local gene delivery of heme oxygenase-1 by adeno-associated virus into osteoarthritic mouse joints exhibiting synovial oxidative stress. Osteoarthritis Cartilage 21: 358-367, 2013.

30. Aparicio-Soto M, Sánchez-Hidalgo M, Cárdeno A Rosillo MÁ, Sánchez-Fidalgo S, Utrilla J, Martín-Lacave I and Alarcón-de-la-Lastra C: Dietary extra virgin olive oil attenuates kidney injury in pristane-induced SLE model via activation of HO-1/Nrf-2 antioxidant pathway and suppression of JAK/STAT, NF- $\kappa B$ and MAPK activation. J Nutr Biochem 27: 278-288, 2016.

31. Clérigues V, Guillén MI, Gomar F and Alcaraz MJ: Haem oxygenase- 1 counteracts the effects of interleukin-1 $\beta$ on inflammatory and senescence markers in cartilage-subchondral bone explants from osteoarthritic patients. Clin Sci (Lond) 122: 239-250, 2012

32. Cheng L, Jin Z, Zhao R, Ren K, Deng C and Yu S: Resveratrol attenuates inflammation and oxidative stress induced by myocardial ischemia-reperfusion injury: Role of Nrf2/ARE pathway. Int J Clin Exp Med 8: 10420-10428, 2015.

(1) 8 This work is licensed under a Creative Commons Attribution-NonCommercial-NoDerivatives 4.0 International (CC BY-NC-ND 4.0) License. 